\title{
Transition delay in a boundary layer flow using active control
}

\author{
Onofrio Semeraro $\dagger$, Shervin Bagheri, Luca Brandt and Dan S. Henningson \\ KTH Mechanics, Linné Flow Centre, Swedish e-Science Research Centre (SeRC), \\ SE-100 44 Stockholm, Sweden
}

\begin{abstract}
(Received 11 March 2013; revised 4 June 2013; accepted 5 June 2013; first published online 15 August 2013)
\end{abstract}

Active linear control is applied to delay the onset of laminar-turbulent transition in the boundary layer over a flat plate. The analysis is carried out by numerical simulations of the nonlinear, transitional regime. A three-dimensional, localized initial condition triggering Tollmien-Schlichting waves of finite amplitude is used to numerically simulate the transition to turbulence. Linear quadratic Gaussian controllers based on reduced-order models of the linearized Navier-Stokes equations are designed, where the wall sensors and the actuators are localized in space. A parametric analysis is carried out in the nonlinear regime, for different disturbance amplitudes, by investigating the effects of the actuation on the flow due to different distributions of the localized actuators along the spanwise direction, different sizes of the actuators and the effort of the controllers. We identify the range of parameters where the controllers are effective and highlight the limits of the device for high amplitudes and strong control action. Despite the fully linear control approach, it is shown that the device is effective in delaying the onset of laminar-turbulent transition in the presence of packets characterized by amplitudes $a \approx 1 \%$ of the free stream velocity at the actuator location. Up to these amplitudes, it is found that a proper choice of the actuators positively affects the performance of the controller. For a transitional case, $a \approx 0.20 \%$, we show a transition delay of $\Delta R e_{x}=3.0 \times 10^{5}$.

Key words: boundary layers, boundary layer control, flow control, instability

\section{Introduction}

This paper considers the control of laminar-turbulent transition in the flat-plate boundary layer triggered by the growth and breakdown of finite-amplitude threedimensional disturbances. In clean environments characterized by low levels $\left(T_{u}<1 \%\right)$ of free stream turbulence (FST), nearly two-dimensional Tollmien-Schlichting (TS) wavepackets are often observed in the boundary layer. The amplitude of these perturbations grows at an exponential rate, and when reaching amplitudes of order $1 \%$ of the free stream velocity a rapid breakdown eventually leads to a turbulent flow. This scenario is referred to as the classic route to transition (Kachanov 1994). 
A different scenario, bypass transition as observed for higher values of $\operatorname{FST}\left(T_{u}>1 \%\right)$, is characterized by the presence of three-dimensional streaks. The amplitude of these streamwise elongated structures grows at an algebraic rate and is approximately one order of magnitude larger than that of the TS waves before the final breakdown (Andersson et al. 2001). From a technological point of view it is of interest to control these routes to transition in order to reduce drag, which in turn may lead to significant savings, for instance, in the operational cost of cargo ships and commercial aircraft.

The combination of control theory tools applied to linear system dates back to the works by Joshi, Speyer \& Kim (1997), Bewley \& Liu (1998) and Cortelezzi et al. (1998). In the following decade, several successful projects were initiated extending these concepts to more complicated configurations; an account of research developments can be found in Kim \& Bewley (2007), Sipp et al. (2010) and Bagheri \& Henningson (2011).

The main idea behind the present investigation is to explore the possibility of delaying transition to turbulence by mitigating the perturbations arising in a flow. To accomplish this aim, linear quadratic Gaussian (LQG) controllers are designed using localized actuators and sensors in combination with reduced-order models (ROMs). We can summarize the procedure as follows.

(a) Introduction of inputs (disturbances and actuators) and outputs (sensors) in the system.

(b) Identification of a ROM.

(c) Design of a low-order controller based on the ROM.

(d) Analysis of the controlled flow by testing the performance with numerical simulations.

In the control literature, this framework is usually referred to as reduce-thendesign (see, e.g., Anderson \& Liu 1989). A ROM properly reproducing the linear input-output behaviour can be obtained in a systematic way using balanced truncation (Moore 1981), usually approximated for high dimensional systems by the snapshotbased algorithm proposed by Rowley (2005); in this case, we need a model of the flow (i.e. the linearized Navier-Stokes equation) and the related adjoint. In a recent application, Ma, Ahuja \& Rowley (2011) propose for fluid flow control a system identification algorithm usually referred to as eigensystem realization algorithm (ERA) (see Juang \& Pappa 1985); they show the equivalence between approximate balanced truncation and ERA, although using the latter algorithm neither simulations of the adjoint system nor a Galerkin projection onto the balanced mode basis are necessary to obtain low-order models. Once the low-order controller is designed, it can be tested in full direct numerical simulations (DNS) or large eddy simulations (LES).

The reduce-then-design scheme has been successfully employed for the control of the cavity flow by Rowley \& Juttijudata (2005), Åkervik et al. (2007) and Barbagallo, Sipp \& Schmid (2009), channel flow by Ilak \& Rowley (2008) and two-dimensional infinitesimal perturbations developing in a boundary layer by Bagheri, Brandt \& Henningson $(2009 a)$. The control of three-dimensional infinitesimal perturbations in a spatially developing boundary layer over a flat plate has been analysed by Semeraro et al. (2011). In the present work, the analysis of the closed loop is extended to fully nonlinear simulations to verify the possibility of delaying the transition to turbulence promoted by growing localized perturbations of finite amplitudes. The initial condition is that computed by Monokrousos et al. (2010) using a time-stepperbased optimization (Schmid 2007). Monokrousos et al. (2010) showed that long optimization times provide an initial condition triggering a wavepacket of TS waves, 

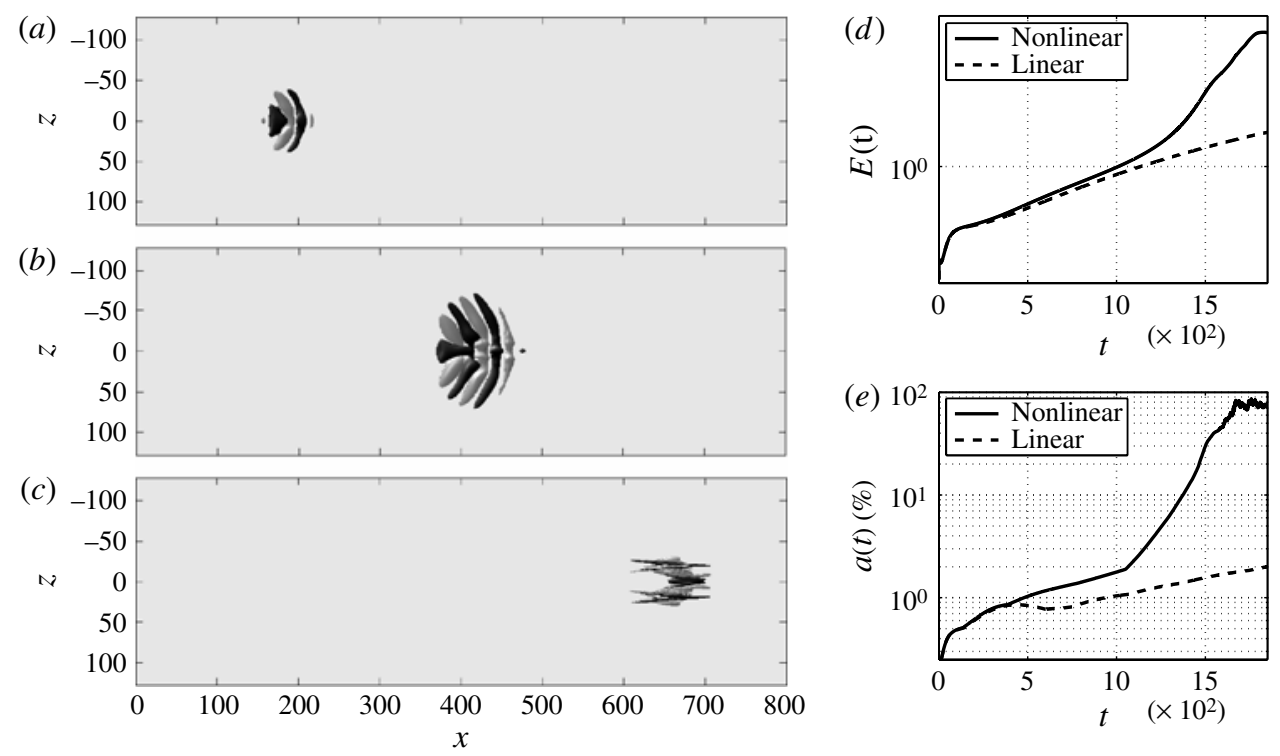

FIGURE 1. Evolution of a TS wavepacket. $(a-c)$ The streamwise component is shown from the top, in the $x-z$ plane, at three different instants of time: $(a) t=400 ;(b) t=1000 ;(c)$ $t=1600$. The isosurface levels equal $30 \%$ of the maximum velocity (grey) and minimum velocity (black). $(d)$ Evolution of the perturbation energy $E(t)$ in time; the dashed line indicates the linear prediction, while the solid line depicts the energy of the packet shown in $(a-c)$. (e) Amplitude $a(t)$ of the wavepacket as a function of time, same legend as $(d)$.

whereas large amplifications in short times are obtained when streamwise vortices are used as initial condition.

We focus our attention on the first type of perturbation, the TS waves. In figure $1(a-c)$, the spatial evolution of the wavepacket is shown from the top, in the $x-z$ plane, at three different instants of time. The Cartesian coordinates are indicated by $\boldsymbol{x}=(x, y, z)$, where $x, y$ and $z$ are the streamwise, wall-normal and spanwise directions. The Reynolds number is defined as $\operatorname{Re}=U_{\infty} \delta_{0}^{*} / \nu$, where $\delta_{0}^{*}$ is the displacement thickness at the inflow position, $U_{\infty}$ is the uniform free stream velocity and $v$ is the kinematic viscosity. We choose $R e=1000$, corresponding to $R e_{x} \approx 3 \times 10^{5}$ at the computational inlet. Hereafter, the perturbation velocity field, defined as the deviation of the velocity components from the stationary baseflow $\boldsymbol{U}(\boldsymbol{x})$, will be denoted as $\boldsymbol{u}(\boldsymbol{x}, t)^{\prime}$. As shown in figure 1(a), the streamwise component of the wavepacket is nearly two-dimensional at $t=400$. This stage of the evolution is well-predicted by linear theory; indeed, the perturbation energy

$$
E(t)=\left\langle\boldsymbol{u}^{\prime}, \boldsymbol{u}^{\prime}\right\rangle_{\Omega},
$$

with $\Omega$ indicating the computational domain, is in good agreement when comparing the fully nonlinear and the linear configurations figure $1(d)$.

The packet grows in size along the spanwise and streamwise directions while propagating downstream in the computational box. At time $t=1000$, the packet starts to develop three-dimensional structures and loses its spanwise coherence. For $t>1000$, a quick energy growth is observed, characterized by a strong deviation from the linear 
prediction. We define the amplitude of a disturbance by

$$
a(t)=\left(\max _{x, y, z} \boldsymbol{u}^{\prime}-\min _{x, y, z} \boldsymbol{u}^{\prime}\right) / 2 U_{\infty}
$$

(see Andersson, Berggren \& Henningson 1999; Andersson et al. 2001). Thus, we account for the maximum and minimum pointwise perturbation velocities. At this stage, the amplitude of the wavepacket quickly increases from $a \approx 1-2 \%$ up to values comparable with the free stream velocity at $t \approx 1600$ (figure $1 e$ ). This final stage is characterized by the presence of a turbulent spot, strongly dominated by three-dimensional structures (see figure $1 c$ ).

In this paper, we examine the effect of the controller on the perturbation when the flow is still laminar, corresponding for the TS waves to amplitudes $a \approx 1 \%$ as shown in figure 1(a), and determine under which conditions the control action can result in a delay of the initial stages of the laminar-turbulent transition. The extension of our previous work is not trivial due to the three-dimensional, localized nature of the control action. The necessity of introducing localized actuators is a constraint often found in experimental configurations and can represent a first limitation of the control design; considering the TS instabilities, applications can be found in the experimental works by Sturzebecher \& Nitsche (2003) and Li \& Gaster (2006). For the bypass transition and the associated streak growth, the reader can refer to the experimental analysis carried out by Lundell (2007) and Monokrousos et al. (2008). In the present investigation, the actuation is produced by a finite number of localized elements described by a solenoidal Gaussian-like function, forcing all of the components of the velocity field. We consider three different configurations of actuators characterized by different distribution of the localized elements in the spanwise direction and different dimension of the forcing. We then move our focus from the analysis of the controllers to the physics of the actuation, which plays a fundamental role in a fully three-dimensional configurations. Indeed, the localization of the actuators can promote instabilities that, in combination with the nonlinearities of the flow, can trigger an early transition to turbulence. Thus, a preliminary parametric analysis is a necessary step for the assessment of the performance that can actually be achieved when a linear controller is used to damp finite-amplitude perturbations.

This paper is organized as follows: we briefly outline the input-output system in $\S 2$. More details about the model reduction problem and the control scheme are given in Appendix. The performance achieved in the nonlinear simulations and the analysis of the controlled system are discussed in $\$ 3$, where the effects of the spanwise distribution of the actuators and of the control effort are investigated. The effects of the actuation are further analysed in $\S 4$ using velocity spectra based on amplitude expansion. The paper ends with a discussion of the main conclusions in $\S 5$.

\section{Configuration}

The dynamics of a viscous, incompressible flow over a flat plate is governed by the Navier-Stokes equations

$$
\begin{gathered}
\frac{\partial \boldsymbol{u}}{\partial t}=-\boldsymbol{u} \cdot \nabla \boldsymbol{u}-\nabla p+\frac{1}{R e} \nabla^{2} \boldsymbol{u}+\lambda(x) \boldsymbol{u}, \\
0=\nabla \cdot \boldsymbol{u},
\end{gathered}
$$

where the velocity field is denoted with $\boldsymbol{u}(\boldsymbol{x}, t)=(u, v, w)$ and the pressure with $p(\boldsymbol{x}, t)$. The simulations presented in this paper were performed using a pseudo-spectral DNS code (Chevalier et al. 2007). The solution is approximated by Fourier expansion in the 


$\begin{array}{lccc} & \text { Cases } & \text { Resolution } & \text { Box } \\ & & N_{x} \times N_{y} \times N_{z} & {\left[L_{x}, L_{y}, L_{z}\right]} \\ \text { Linear } & \text { Control design } & 768 \times 101 \times 120 & {[1000,30,120 \pi]} \\ \text { Nonlinear } & \text { Parametric study } & 768 \times 101 \times 256 & {[1000,30,256]} \\ \text { Nonlinear } & \text { Transition delay } & 1536 \times 101 \times 384 & {[2000,30,256]}\end{array}$

TABLE 1. Summary of the performed simulations. For each case, the resolution and the computational box are reported.

horizontal directions $(x, z)$ and Chebyshev polynomials along the wall-normal direction $(y)$; the temporal advancement is approximated by a four-step third-order Runge-Kutta method for the nonlinear term, and a second-order Crank-Nicolson method for the linear term. Thus, within the spatial approximation, periodicity is assumed in the spanwise direction and enforced in the streamwise direction by a fringe region $\lambda(x)$ at the outlet of the domain (Nordström, Nordin \& Henningson 1999). The fringe region extends for $20 \%$ of the physical length of the domain in all of the cases summarized in table 1. Dirichlet conditions enforce zero perturbations velocity at the wall $(y=0)$, while Neumann conditions are applied in the free stream $\left(y=L_{y}\right)$.

Linear simulations were performed for the design of the controller. Simulations of the nonlinear flow were used for the assessment of the controller performance in presence of finite-amplitude perturbations. The box size and resolution are reported in table 1 and discussed in the following sections.

\subsection{Inputs and outputs}

The control design is based on the Navier-Stokes equations linearized around a steady state, $\boldsymbol{U}(\boldsymbol{x})=(U, V, 0)$; the baseflow is computed by marching forward in time the discretized fully nonlinear (2.1) until a steady solution is obtained. In the following, the problem variables will indicate the spatial-discrete counterparts, while using the same symbols of the spatial-continuous variables.

The set-up is sketched in figure 2, where we display the arrays of actuators and sensors, spanning the spanwise direction. The arrays are formed by $m$ localized actuators and $p$ sensors; as shown in the sketch, the disturbance is introduced far upstream in the domain. The controller is designed such that the perturbation energy is minimized in the region downstream of the actuators. The resulting linear-time invariant system is

$$
\begin{aligned}
\dot{\boldsymbol{u}}(t)^{\prime} & =\boldsymbol{A} \boldsymbol{u}(t)^{\prime}+\boldsymbol{B}_{1} \boldsymbol{w}(t)+\boldsymbol{B}_{2} \phi(t), \\
\psi(t) & =\boldsymbol{C}_{2} \boldsymbol{u}(t)^{\prime}+\eta \boldsymbol{g}(t), \\
\boldsymbol{z}(t) & =\boldsymbol{C}_{1} \boldsymbol{u}(t)^{\prime}
\end{aligned}
$$

The perturbation velocity is indicated with $\boldsymbol{u}(t)^{\prime}=\left(u^{\prime}, v^{\prime}, w^{\prime}\right) \in \mathbb{R}^{n}$, where the $u^{\prime}, v^{\prime}$ and $w^{\prime}$ are the streamwise, wall-normal and spanwise components. The number of degree of freedom resulting from the discretization of the system is $n=3 N_{x} N_{y} N_{z}$, i.e. the number of grid points times the velocity components. The system matrix $\boldsymbol{A} \in \mathbb{R}^{(n \times n)}$ represents the linearized and discretized Navier-Stokes equations including the boundary conditions, already reported above. The column vector $\boldsymbol{B}_{1} \in \mathbb{R}^{n}$ models incoming external perturbation, located far upstream (on the left in figure 2). 


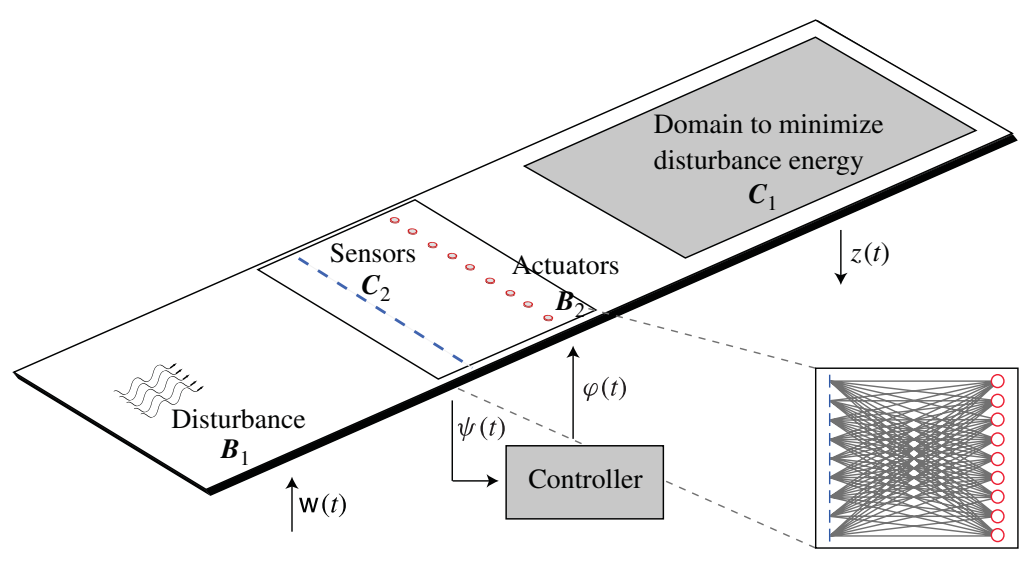

FIGURE 2. (Colour online) Sketch of the control configuration. The disturbance $\left(\boldsymbol{B}_{1}\right)$ consists of an optimal initial condition. A low-order LQG controller is designed; based on the output extracted by a spanwise row of sensors $\left(\boldsymbol{C}_{2}\right)$, the controller computes the control signals feeding a row of actuators $\left(\boldsymbol{B}_{2}\right)$. The objective of the controller is to minimize the disturbance energy in the region marked with the grey colour. The inset figure shows that all of the sensors are wired to all of the actuators (so-called centralized control, see Appendix).

The optimal, localized initial condition triggers a wavepacket of TS waves (Monokrousos et al. 2010). The temporal behaviour described by the signal $\boldsymbol{w}(t) \in \mathbb{R}$ is the disturbance signal excitation, assumed as a unit-variance white noise process. The matrix $\boldsymbol{B}_{2} \in \mathbb{R}^{n \times m}$ represents the spatial distribution of the actuators, located in a spanwise array of $m$ elements and modelled as volume forcing localized close to the walls. The control signal is represented by $\phi(t) \in \mathbb{R}^{m}$.

The output signals $\psi(t) \in \mathbb{R}^{p}$ in $(2.2 b)$ are extracted by the array of $p$ localized sensors $\boldsymbol{C}_{2} \in \mathbb{R}^{p \times n}$, which are placed a short distance upstream of the actuators. The noise contamination of the signal is modelled by the unit-variance white noise $\boldsymbol{g}(t) \in \mathbb{R}^{p}$, whose level is set by the constant $\eta$. A large value of $\eta$ introduces high levels of noise corruption on the measurements $\psi(t)$, while a small value indicates high fidelity of the information extracted by the sensors $\boldsymbol{C}_{2}$.

The output signal $\boldsymbol{z}(t) \in \mathbb{R}^{k}$ in $(2.2 c)$ is used to assess the performance of the controller. Output projection is performed (Rowley 2005): the signal is obtained by projecting the velocity field on a sequence of $k=10$ proper orthogonal decomposition (POD) modes, capturing $\approx 93 \%$ of the perturbation energy. The modes are generated by a dataset of snapshots collected from the impulse response to the initial condition $\boldsymbol{B}_{1}$ and are represented by the rows of $\boldsymbol{C}_{1} \in \mathbb{R}^{k \times n}$. By adopting this approach, the signal $\boldsymbol{z}(t) \in \mathbb{R}^{k}$ represents an approximation of the energy content of the system; based on the output $\boldsymbol{z}(t)$, a fictitious output is defined as

$$
\boldsymbol{z}(t)^{\prime}=\left[\begin{array}{c}
\boldsymbol{C}_{1} \\
\mathbf{0}
\end{array}\right] \boldsymbol{u}(t)^{\prime}+\left[\begin{array}{c}
\boldsymbol{0} \\
\boldsymbol{R}^{1 / 2}
\end{array}\right] \phi(t),
$$

where the diagonal entries of the matrix $\boldsymbol{R} \in \mathbb{R}^{m \times m}$ correspond to the control penalty $l^{2}$. Thus, the first term is related to the energy of the system through the projection performed on the POD basis, while the second term is related to the control effort. 


\begin{tabular}{lccccccc} 
Configuration & ROM size & Spacing & Sensors & \multicolumn{4}{c}{ Actuators } \\
& $r$ & $\Delta z$ & $p$ & $m$ & $\sigma_{x}$ & $\sigma_{y}$ & $\sigma_{z}$ \\
L9 & 60 & 20 & 9 & 9 & 5 & 1.5 & 6 \\
L17 & 200 & 10 & 17 & 17 & 5 & 1.5 & 6 \\
S17 & 150 & 10 & 17 & 17 & 2 & 1.5 & 4
\end{tabular}

TABLE 2. The main parameters characterizing the three configurations used in this work: the size of ROM $(r)$; the spacing among each element of the sensors' and actuators' array $(\Delta z)$; the number of sensors $(p)$ and actuators $(m)$; the spatial distribution of the actuators (described by $\sigma_{x}, \sigma_{y}, \sigma_{z}$ ). In all of the configurations, the central element of the sensors and actuators arrays is placed at $z=0$. The sensors are characterized by the same spatial distribution for all of the cases $\left(\sigma_{x}, \sigma_{y}, \sigma_{z}\right)=[5,1.5,6]$

A quadratic cost function is defined via (2.3):

$$
\begin{aligned}
\mathscr{E}\left\{\left\|\boldsymbol{z}(t)^{\prime}\right\|_{L^{2}(0, \infty)}^{2}\right\} & =\mathscr{E}\left\{\frac{1}{2} \int_{0}^{\infty}\left(\boldsymbol{z}(t)^{\prime}\right)^{H} \boldsymbol{z}(t)^{\prime} \mathrm{d} t\right\} \\
& =\mathscr{E}\left\{\frac{1}{2} \int_{0}^{\infty}\left(\boldsymbol{u}(t)^{\prime}\right)^{H} \boldsymbol{C}_{1}^{H} \boldsymbol{C}_{1} \boldsymbol{u}(t)^{\prime}+\phi(t)^{H} \boldsymbol{R} \phi(t) \mathrm{d} t\right\},
\end{aligned}
$$

where $\mathscr{E}(\cdot)$ is the expectation operator and the superscript $(\cdot)^{H}$ indicates the transpose matrix. The aim of the controller is to minimize (2.4) by computing a control signal $\phi(t)$ based on the information obtained from the signals $\psi(t)$. The control effort can be modified by changing the parameter $l$ : large values penalize the control signal, and are characterized by a weaker actuation, while higher control efforts are obtained when low values for $l$ are chosen.

Each of the $m$ actuators in $\boldsymbol{B}_{2}$ and $p$ sensors in $\boldsymbol{C}_{2}$ is represented by a volume forcing localized in a region close to the wall. In particular, the columns of the matrix $\boldsymbol{B}_{2}$, and the rows of the matrix $\boldsymbol{C}_{2}$ are obtained from the discretization of the Gaussian-like solenoidal function

$$
\boldsymbol{h}\left(\boldsymbol{x}, \boldsymbol{x}_{0}\right)=\left(\begin{array}{c}
\sigma_{x} \hat{y} \\
-\sigma_{y} \hat{x} \\
0
\end{array}\right) \exp \left(-\hat{x}^{2}-\hat{y}^{2}-\hat{z}^{2}\right)
$$

where

$$
\hat{x}=\frac{x-x_{0}}{\sigma_{x}}, \quad \hat{y}=\frac{y}{\sigma_{y}}, \quad \hat{z}=\frac{z-z_{0}}{\sigma_{z}} .
$$

All of the elements of the arrays have the same spatial distribution; the scalar quantities $\sigma_{x}, \sigma_{y}$ and $\sigma_{z}$ determine the size of the inputs and are listed in table 2 . The location in the $x-z$ plane is given by $\boldsymbol{x}_{0}=\left(x_{0}, z_{0}\right)$. From this, we define each actuator as

$$
\boldsymbol{B}_{2 j}=\boldsymbol{h}\left(\boldsymbol{x}, \boldsymbol{x}_{\phi j}\right),
$$

where $\boldsymbol{x}_{\phi j}=\left(400, z_{\phi j}\right)$. The location of the elements along the spanwise direction $z$ is given by

$$
z_{\phi j}=j \Delta z, \quad j=-(m-1) / 2,-(m-1) / 2+1, \ldots,(m-1) / 2,
$$


where $\Delta z$ is the spacing among the actuators along the spanwise direction and $j$ an integer number (see also table 2). From a physical point of view, the actuators mimic a manipulation of the flow close to the wall. A sketch of the actuators' array is shown in figure 2; the number of actuators $m$ for each array and the spacing among the elements are two of the main parameters analysed in $\S 3.1$. The flow measurements are obtained by averaging the velocity field over a small portion of the domain defined by the function (2.5) used as weights. In particular,

$$
\boldsymbol{C}_{2 j} \boldsymbol{u}(t)^{\prime}=\int_{\Omega}\left(\boldsymbol{h}\left(\boldsymbol{x}, \boldsymbol{x}_{\psi j}\right)^{H} \boldsymbol{u}(t)^{\prime}\right) \mathrm{d} x \mathrm{~d} y \mathrm{~d} z,
$$

with $\boldsymbol{x}_{\psi j}=\left(300, z_{\psi j}\right)$. The distribution along the spanwise direction $z$ varies according to (2.8), where $p=m$. The number of sensors $p$ characterizing each configuration is reported in table 2; note that the spatial size of the weights is kept equal for all of the cases, with $\left(\sigma_{x}, \sigma_{y}, \sigma_{z}\right)=[5,1.5,6]$. Finally, as mentioned before, the weights for the output $\boldsymbol{z}(t)$ are represented by the POD modes.

Our choice of model for the actuators and sensors as localized volume forcing is relevant for practical implementations. Examples are provided by the experimental works by Sturzebecher \& Nitsche (2003) and Li \& Gaster (2006) for control of TS wavepackets. Moreover, the actuator chosen here makes the setup rather close to the action provided on the flow by simple models mimicking plasma actuators (Kriegseis 2011). Indeed, a first simplified way to model them consists of localized forcing which reproduce the net force generated by the actuator. These actuators have recently received considerable attention as a viable candidate for transition control (Grundmann \& Tropea 2008).

The control design follows the same steps outlined in Semeraro et al. (2011), where the control of three-dimensional perturbations was investigated within the linear framework. We follow the reduce-then-design scheme (see e.g. Anderson \& Liu 1989); first, ROMs of the system are obtained by applying the ERA, see Juang \& Pappa (1985) and Ma et al. (2011). The control design is performed by following the LQG approach, applied on the low-order models; for a derivation of the LQG solution, we refer the reader to Lewis \& Syrmos (1995) and Dullerud \& Paganini (1999). More details on the control design are contained in Appendix.

The multi-input multi-output (MIMO) configuration characterizing the system requires a proper multivariable approach for the control design (see e.g. Skogestad \& Postlethwaite 2005). In this work, we followed the centralized control approach, where all of the $m$ actuators are connected to all the $p$ sensors and only one control unit is designed. For centralized controllers, the stability of the closed-loop system is guaranteed (Glad \& Ljung 2001).

\section{Transition delay}

In this section, the controlled flow is analysed; first, in $\S 3.1$, the performance is discussed by comparing the three different configurations listed in table 2 within the linear approximation. The analysis is completed within the nonlinear framework by investigating the effect of the controller when finite-amplitude perturbations are considered (\$3.2). We identify the range of amplitudes where the controllers are effective; to this end, we introduce a global measure that accounts for the energy damping. Finally, the delay of transition from laminar to turbulent flow is investigated by considering a longer box in $\S 3.3$. A summary of the simulations performed is reported in table 1 . 


\subsection{Performance in the linear regime}

A rigourous assessment of the performance within the linear limit has been carried out by considering a parametric analysis; we investigate two different sets of parameters:

(1) the parameters characterizing the hardware of the controllers, i.e. the location along the streamwise direction of the actuator and sensor rows, the spacing among the localized elements of each row and the size of the actuators and sensors;

(2) the control penalty $l$; we assume the noise level $\eta=0.01$ fixed.

In particular, in addition to the configuration analysed in Semeraro et al. (2011), hereafter labelled as $L 9$, two new setups are introduced, labelled as $L 17$ and $S 17$ (see table 2).

The control unit $L 9$ consists of elements equispaced along $z$ with $\Delta z=20$; this spacing has deemed sufficient in Semeraro et al. (2011) to guarantee good performance in the linear regime. The number of actuators $m=9$ is chosen to cover the width of the travelling TS waves at the actuators' location. The elements are the Gaussian-like functions in (2.5), whose spatial shape is defined by the values of $\sigma_{x}, \sigma_{y}$ and $\sigma_{z}$ (reported in table 2). The same distribution is considered for the elements of the two arrays used in $L 17$; in this case, $m=p=17$ elements are introduced for each array, equispaced with $\Delta z=10$. Thus, we consider an array covering the same spanwise length of the controller $L 9$, but characterized by half of the original spacing. Finally, $S 17$ is based on actuators with a smaller spatial support. In this configuration, we have chosen the same number of elements and same distribution of the controller $L 17$ along the spanwise direction, i.e. $m=p=17$ and $\Delta z=10$. The aim is to evaluate how the local shape of the actuators can influence the actual performance of the controller; for the controller $S 17$, the shape of the actuators is elongated along the spanwise direction. These choices and the effects on the performance are shown in this section and thoroughly discussed in $\S 4$ by analysing the flow after the actuation.

Note that effective ROMs with a small number of actuators and sensors, $m=p<10$, were obtained by using only $r=60$ modes, while $r=200$ and $r=150$ modes were necessary to properly reproduce the input-output dynamics for the cases $L 17$ and $S 17$, respectively. Convergence tests were performed by varying the sampling parameters of the outputs extracted from the system; more details are provided in Appendix.

As already mentioned, all of the configurations are characterized by actuators placed at $x_{\phi}=400$ and sensors $\boldsymbol{C}_{2}$ placed at $x_{\psi}=300$. This choice is based on the knowledge of the boundary layer instabilities. The actuator location can be chosen without any restriction along the streamwise direction; indeed, the region of highest sensitivity to perturbation extends over all of this area as shown by Brandt et al. (2011). Once the actuators are placed, the location of the sensor can be chosen according to the control strategy. Here, the row of estimation sensors $\boldsymbol{C}_{2}$ is upstream of the row of actuators. This specific configuration dynamically corresponds to a disturbance feedforward controller, a special case of output feedback control within the framework formalized by Doyle et al. (1989). Indeed, due to the presence of strong time delays in the system, induced by the convective nature of the boundary layer, the dynamics from the actuators to the sensors is negligible; therefore, the action of the controller is not fed back by the sensors.

This configuration guarantees the best performance in terms of perturbation energy damping as shown by Belson et al. (2013) for the corresponding two-dimensional case. An analogous result is presented by Juillet, Schmid \& Huerre (2013), where it is shown that the feedforward approach is equivalent to an optimal LQG for convective flows and for sensors placed sufficiently far upstream of the actuators. In a 


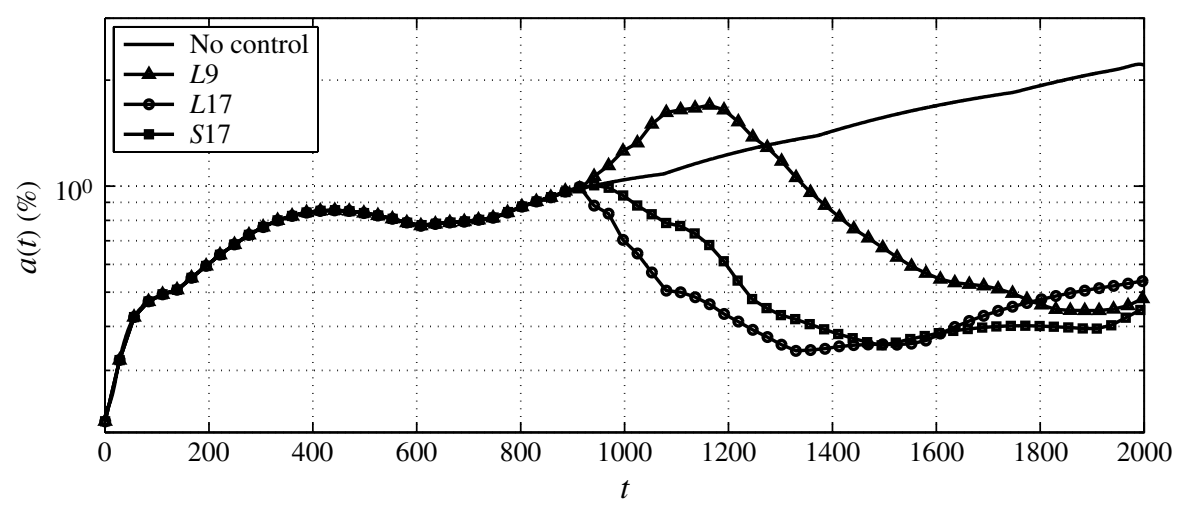

FIGURE 3. Perturbation amplitude $a(t)$ as a function of time $t$. The uncontrolled case (solid black line) is compared with three different controlled systems: $L 9(-\triangle-), L 17(-\bigcirc-)$ and $S 17(-\square-)$.

feedforward controller the stability of the closed-loop is mostly related to the quality of the ROM and its ability to reproduce the input-output behaviour of the system. However, despite the advantages in terms of performance and stability, off-design conditions are potentially critical due to possible lack of robustness of the resulting controller to the uncertainties of the system.

The amplitudes of the perturbation $a(t)$, defined in (1.2), are shown in figure 3 . Linear simulations are performed (see table 1); the resolution was deemed sufficient to resolve the action of the controller on the flow. The three configurations are compared by choosing the control penalty $l$ giving the best performance in the range $l \in[1,400]$. In particular, $l=150$ for the controllers $L 9$ and $L 17$, while $l=100$ for $S 17$.

Not surprisingly, actuators separated with a smaller $\Delta z$ improve the performance of the controller. Indeed, when the controller $L 9$ is applied, an increase of $a(t)$ can be observed at $t>800$. Only later in time, when $t>1200$, it is possible to observe a quick decrease of the amplitudes with respect to the uncontrolled case. Note that the controller is always active. However, due to the spatial localization of the wavepackets and the convective nature of the flow, the wavepacket traverses the actuators' array at $t \in[800,1200]$; thus, the amplitude increase is the footprint of the action of the controller L9. This behaviour is not observed when $L 17$ and $S 17$ are used; indeed, the action of these controllers is characterized by a smoother damping of the amplitudes. At a later time, corresponding to locations further downstream of the actuators, we observe for $L 17$ and $S 17$ a renewed growth of the disturbance amplitude $a(t)$. From the physical point of view, this behaviour is mainly connected to the action of the localized elements on the original TS wave structure. When the configuration $L 9$ is used, the structure is distorted to a fully three-dimensional structure; the resulting structure propagating downstream is characterized by a quick decay, see figure 3. Only at the end of the computational domain, corresponding to $t>1800$, it is possible to observe a slow increase of the $a(t)$ also for $L 9$. Indeed, in a longer computational box a new growth can be observed, due to the recovering of the original two-dimensional structure of the TS wave (see $\S 3.3$ ).

In conclusion, configurations with a larger number of actuators ( $L 17$ and $S 17$ ) quench more efficiently the TS, but are also characterized by a faster recovering of the two-dimensional coherence of the TS waves structure along the $z$ coordinate. The 
three-dimensional structure of the perturbations modified by the action of the actuators is further investigated in $\S \S 3.3$ and 4 .

\subsection{Parametric analysis in the nonlinear regime}

We focus our attention on the action of a linear controller when finite-amplitude disturbances are considered. A parametric analysis has been carried out by using numerical simulations of the controlled flow in the nonlinear regime. The computational box and the resolution are reported in table 1. LES were performed using the ADM-RT subgrid-scale model (SGS) for the simulation of transition. The model employs the relaxation term (RT) proposed in the context of the approximate deconvolution model (ADM) by Adams \& Stolz (1999). It has been shown by Schlatter, Stolz \& Kleiser (2004) that the ADM-RT model is accurate and robust in predicting transitional and turbulent flows with spectral methods. The LES resolution was validate against full DNS checking the location/time of the transition to turbulence. Note that the SGS term is effectively active only when the flow becomes fully nonlinear.

We focus on the actuators' action on the flow and their effects in the nonlinear regime. To this aim, we concentrate our analysis on the number of actuators $m$, the spanwise distribution $\Delta z$, the actuator size (defined with $\sigma_{x}, \sigma_{y}$ and $\sigma_{z}$ in (2.5)), the control penalty $l$ and the amplitude in (1.2).

Starting from the knowledge of the boundary layer instabilities and assuming that a strong reduction in energy can lead to an actual delay of the transition to turbulence, we define a global measure of the controller performance

$$
\overline{\Delta E}=\log _{10}\left(\frac{1}{T} \int_{0}^{T} \frac{E_{c}(t)}{E(t)} \mathrm{d} t\right) .
$$

In the above expression, $E(t)$ is the energy (1.1) of the uncontrolled flow and $E_{c}(t)$ the same quantity for the controlled flow; in the parameter $\overline{\Delta E}$, we consider the ratio between the two quantities. This is averaged in the interval $t=[0, T]$, where $T=2000$; at this time, the wavepacket reaches the end of the physical domain at $x=800$ and leaves the computational box. Note that the value of maximum damping of the energy perturbation is underestimated, because the averaged value includes also the interval when the wavepacket is still upstream of the actuators $(t<800)$. Negative values $\overline{\Delta E}<0$ correspond to damping of the perturbation energy, while for positive values $\overline{\Delta E}>0$ the controller triggers new instabilities that lead to a quick increase of the energy (see $\S 4$ ). The quantity $\overline{\Delta E}$ is shown for different values of the reference amplitude $a_{\text {ref }}$ and the control penalty $l$ in figure $4(a-c)$ for the three different configurations in table 2 . We choose the reference amplitude at the actuator location $x_{\phi}=400$. The leading edge of the wavepacket approaches this location at $t \approx 800$; thus, the amplitude of the uncontrolled wavepacket $a_{r e f}=a(800)$ is taken as a reference value.

The range of values $a_{r e f} \in[0.09,2.40] \%$ is investigated and reported along the $y$-axis in figure $4(a-c)$. The performances achieved in the linear case are reported as the lowest value along the $y$-axis of the maps in figure $4(a-c)$. The $x$-axis spans the control penalty range $l \in[100,400]$.

An alternative choice of measure is the energy distortion $E(t)_{c}-E(t)$; we verified that the resulting map is equivalent with that obtained with $\overline{\Delta E}$ in (3.1). The norm $\|\boldsymbol{z}\|$ can be used in replacement of the perturbation energy $E(t)$ in the ratio appearing in (3.1). Although the quantity in (3.1) does not provide a measure of the delay of the 

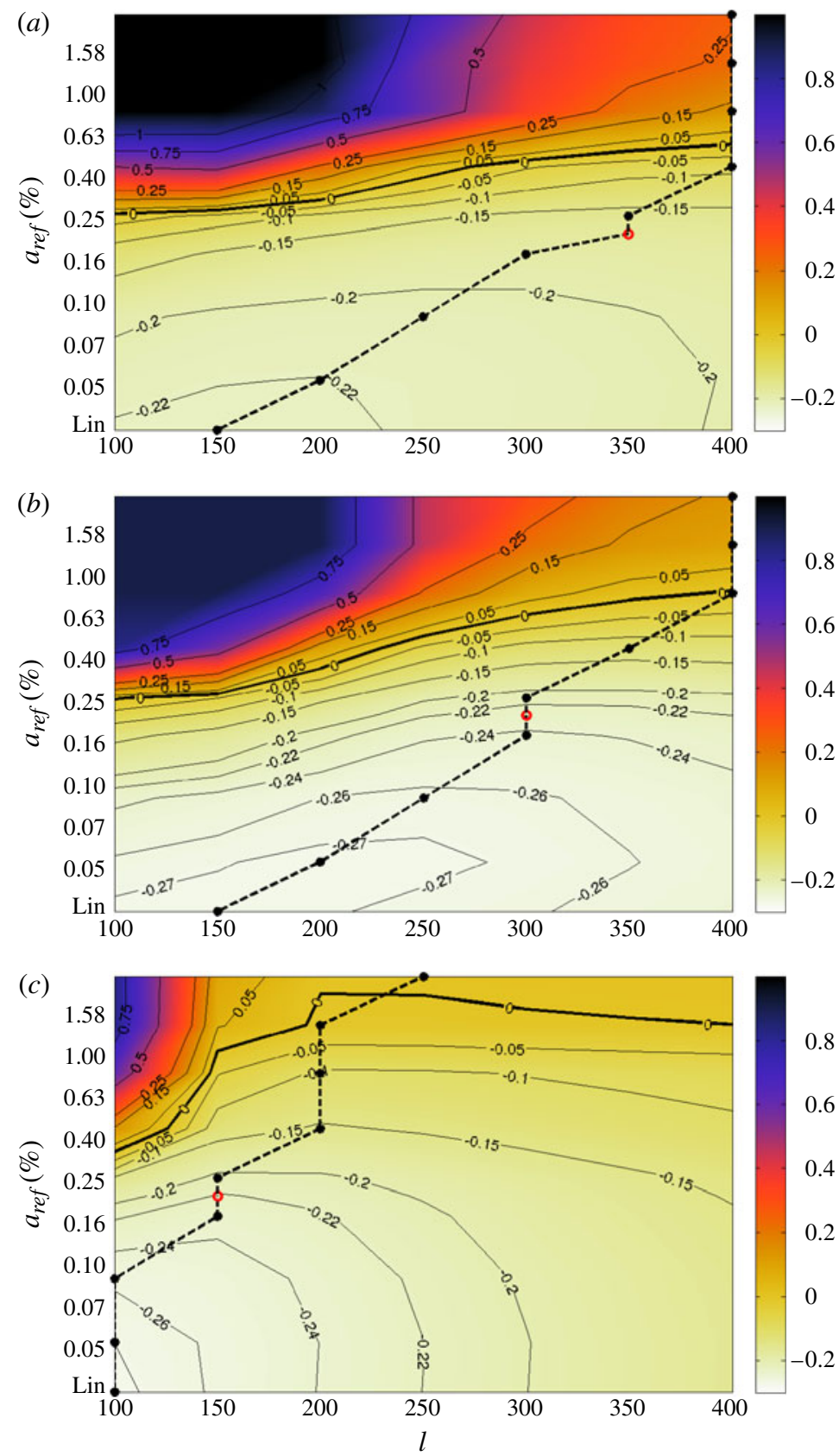

FIGURE 4. The performances of the controllers $L 9(a), L 17(b)$ and $S 17(c)$ are compared for different control penalties $l$ and reference amplitude $a_{\text {ref }}$. The maps depict $\overline{\Delta E}$, given by (3.1). The value $\overline{\Delta E}=0$ marks two regions in each map: in the lower area, where $\overline{\Delta E}<0$, the controllers are effective; when $\overline{\Delta E}>0$, controllers do not provide a correct action. The dashed line indicates the value $l$ corresponding to the best performance for each $a_{r e f}$. The red dots indicate the values $\left(l, a_{r e f}\right)$ used for the analysis of the transition delay in $\S 3.3$. 
transition to turbulence, we assume that a drastic reduction of the perturbation energy results in a delay of the transition processes.

As shown in figure $4(a)$, for the disturbance amplitudes $a_{\text {ref }} \approx 0.25 \%$ the controller $L 9$ results in $\overline{\Delta E}<0$. Note that in this range, the best performance is achieved for values of $l$ that are progressively higher (for instance, compare the linear limit $l=150$, with the $l$ at $a_{\text {ref }} \approx 0.25 \%$ ). Thus, the best value for the control penalty $l$ increases with the amplitudes. This is in contrast to the linear setting, where a smaller control cost results in a better control performance. The trend can be attributed to the fact that high actuation amplitudes trigger nonlinear effects more quickly than low amplitudes.

A similar map characterizes the controller $L 17$ (figure $4 b$ ), where the spacing among the elements in the spanwise direction is $\Delta z=10$. However, considering the contour level of $\overline{\Delta E}\left(a_{r e f}, l\right)$, it is possible to note an improvement of the overall performance. Also for this case, we observe that higher values of the control penalty $l$ are needed when higher amplitudes are considered.

Finally, the performance of the configuration $S 17$ is analysed in figure $4(c)$. The different layout of the actuators, characterized now by a smaller spatial distribution, $m=17$ and $\Delta z=10$, results in a significant improvement of the performance. The operating region extends up to $a_{\text {ref }} \approx 1.50 \%$; in the range $0.40 \%<a_{\text {ref }}<1.50 \%$ we can identify an interval of values $l>150$ where the controller is still effective; vice versa, a stronger control action $(l<150)$ results in a quick decay of the performance and possibly early transition to turbulence. Note that in this same range of amplitudes for the controllers $L 9$ and $L 17$ can not lead to an energy damping. The performance of the configuration $S 17$ gradually decays when increasing the amplitude up to $a_{r e f} \approx 1.50 \%$, until the controller is not effective anymore and secondary instabilities promote an increase of the perturbation energy.

Note that the performance of the controller $S 17$ is characterized by a good agreement with the linear prediction up to $a_{\text {ref }} \approx 0.10 \%$; this is not the case for the controllers $L 9$ and $L 17$ (figure $4 a-b$ ). This improvement is mainly related to a reduced effect of the three-dimensional action of the controller $S 17$ on the nonlinearities of the flow (see $\S 4$ ).

In conclusion, we observe that the actuation has a strong influence on the achievable performance of the optimal controllers designed for our configuration. In particular, what is significant is the role of the spatial distribution of the localized forcing and its influence when higher amplitudes are considered. A different spatial distribution of the actuator forcing, characterized by a smaller spatial support, results in a controller more effective over a wider range of amplitudes.

\subsection{Transition delay}

In this section, we explore the possibility of using the controllers to delay the transition to turbulence. We consider long computational box $\left[L_{x}, L_{y}, L_{z}\right]=$ $\{2000,30,256\}$, to reach turbulence before the outflow, while having a laminar flow at the actuator location. For a qualitative analysis of the results, we refer to figure 5 where the time evolution of the amplitude of a TS wavepacket with $a_{\text {ref }}=0.21 \%$ is shown; the control penalty is chosen by considering the parametric analysis in figure $4(a-c)$. The uncontrolled wavepacket is depicted at $t=1000$ and $t=1500$ in figure $5(e, b)$, respectively. The wavepacket is still clearly laminar and extends in the spanwise direction while propagating downstream. This stage of the evolution is characterized by exponential growth until the disturbance breakdowns to a fully three-dimensional structure, where higher wavenumbers are quickly triggered; for the chosen amplitude, a turbulent spot is observed in the computational box at $t>3000$. 


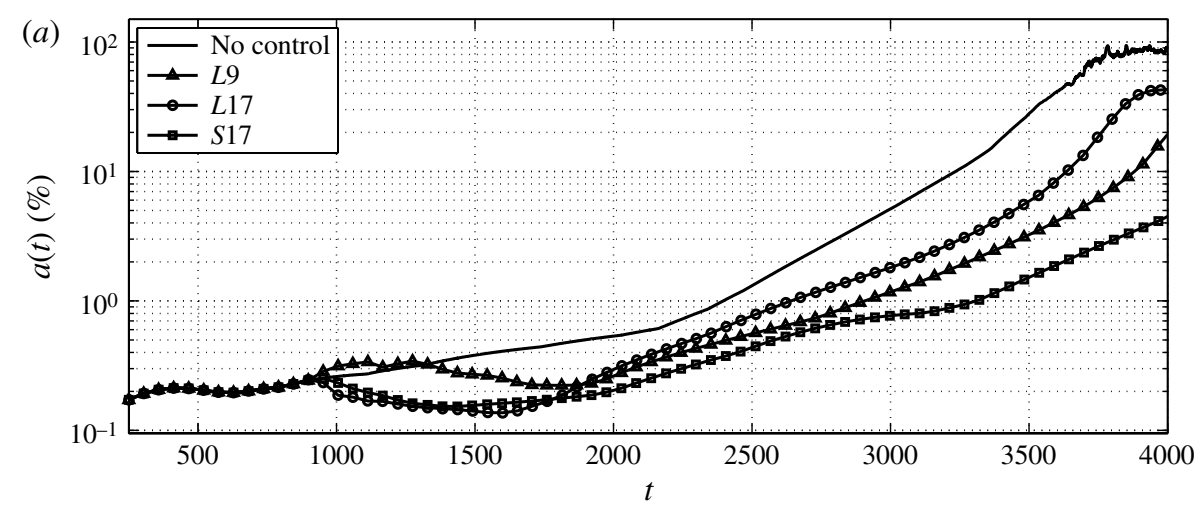

(b)

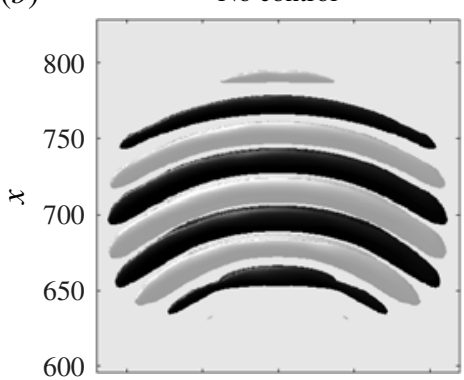

(c) Control, $L 9$

(d) Control, $S 17$

(e)
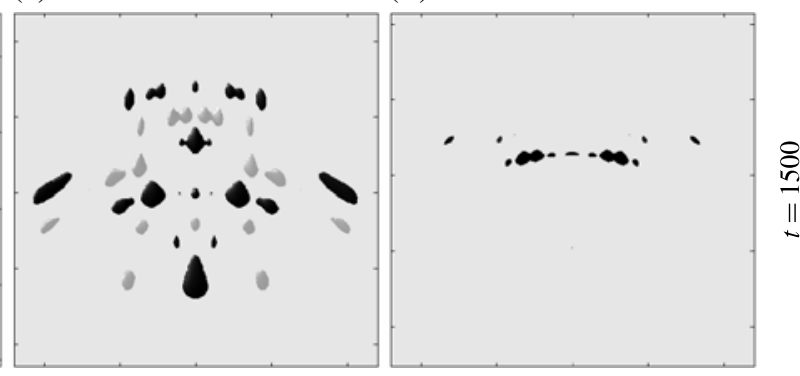

$(f)$

$(g)$
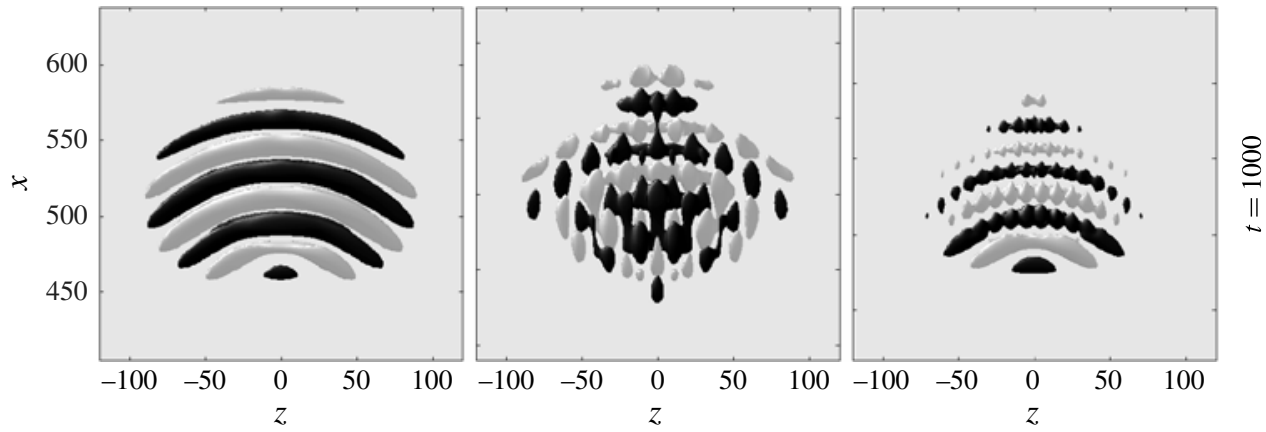

FIGURE 5. Spatial and temporal evolution of a TS wavepacket. In (a) the black solid line depicts the evolution in time of the amplitude $a(t)$ for the uncontrolled case. The lines with markers show $a(t)$ when the control is active for the cases in table 3: $L 9(-\triangle-), L 17(-\bigcirc-)$ and $S 17(-\square-)$. Frames $(b-g)$ show instances from the flow field; the isosurface corresponds to $30 \%$ of the maximum of the streamwise velocity component (grey) and minimum velocity (black) of the corresponding uncontrolled case. The packets are shown from the top, in the $x-z$ plane; the direction of the flow is oriented in the upright direction. The uncontrolled configuration is shown in the leftmost frames $(e)$ and $(b)$, corresponding to $t=1000$ and $t=1500$, respectively. For the same time instants, the action of the controller $L 9$ is shown in the middle frames $(f)$ and $(c)$, while the rightmost frames $(g)$ and $(d)$ depict the action of the controller $S 17$. 


\begin{tabular}{lllll} 
Case & Penalty & \multicolumn{3}{c}{ Transition delay } \\
& $l$ & $\Delta x$ & $\Delta x / L_{x}$ & $\Delta R e_{x}$ \\
$L 9$ & 350 & 250 & $15 \%$ & $2.5 \times 10^{5}$ \\
$L 17$ & 300 & 200 & $12 \%$ & $2.0 \times 10^{5}$ \\
$S 17$ & 150 & 300 & $18 \%$ & $3.0 \times 10^{5}$
\end{tabular}

TABLE 3. Test cases for the analysis of the transition delay. The performance is compared considering the achieved transition delay in terms of $\Delta x$, the ratio $\Delta x / L_{x}$ and $\Delta R e_{x}$. Here, $L_{x}=1700$ corresponds to the physical length of the domain along the streamwise direction, i.e. without considering the fringe region.

The amplitude curves when the control is active are shown in the same figure with marked solid lines; the three configurations introduced in table 2 are compared. For each of them, the optimal control penalty $l$ is chosen according to the results of the parametric analysis in figure 4; these values are reported in table 3 .

As already observed in figure 3 , the action of controller $L 9$ is characterized by an initial growth of the amplitude when the actuator is active. This initial increase combined with the nonlinearities of the flow is responsible of the worsening of performance; indeed, by comparing figures 3 and $5(a)$ in the interval $t \in[0,2000]$, we observe a deviation from the linear behaviour and a less efficient damping of the perturbation energy. The presence of higher amplitudes in the controlled flow is observed to be crucial in the nonlinear simulations and represents a limitation for the performance of the controller.

The best performance is achieved for the configuration $S 17$ in terms of amplitude damping and transition delay. The disturbance traverses the array of actuators after approximately 1000 time units. Since the action of the controller is limited only to the region close to the actuators, where the flow is still locally unstable, the perturbation begins to grow again further downstream, corresponding to $t \approx 1500$ and eventually triggers transition. In this case, we observe a smoother damping of the amplitude during the initial phase of the control action. Comparing the spatial structures of the disturbance with and without control in figure $5(b-g)$, we observe that the controller changes the structure of the perturbation. The behaviour of the controllers strongly differs. When $m=9$, the original nearly two-dimensional wavepacket is sliced into a more complicated three-dimensional structure as it traverses the actuators, as shown at $t=1000$ and $t=1500$ in the insets $f$ and $c$. In a purely linear analysis a three-dimensional wavepacket is less unstable (i.e. has a smaller growth rate), but in a nonlinear regime it requires a lower threshold to trigger turbulence (Schmid \& Henningson 2001). To achieve transition delay, it is therefore essential that the controller $L 9$ acts in the linear regime of the transition process. When a shorter distance among the actuator is chosen, $\Delta z=10$ as in $S 17$, the structure of the wavepacket is not characterized by strong three-dimensionality (compare figure $5 f, g$ ); indeed, the structure preserves the two-dimensional coherence along the spanwise direction. Within the linear framework, this feature makes the controller less efficient than the $L 9$ setup, (see §3.1). However, the controller action is less influenced by the nonlinear effects and closer to the linear prediction at higher amplitudes. In other words, shorter spanwise scales are more beneficial to delay transition as they introduce less dangerous perturbations. This effect is achieved by combining a smaller $\Delta z$ with a smaller width of the distribution of the volume forcing. Further downstream of 


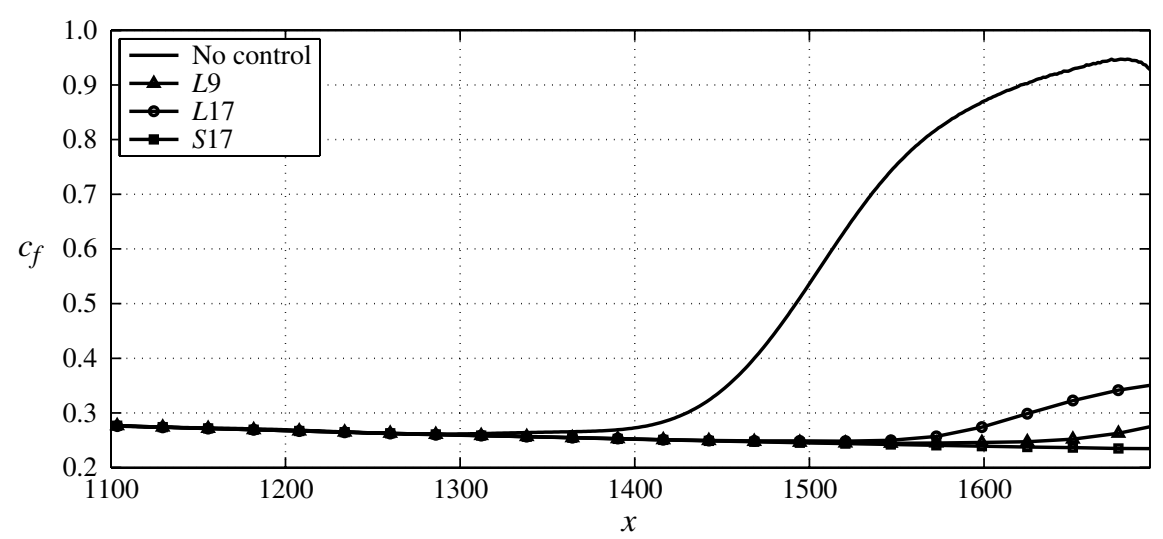

FIGURE 6. The skin friction coefficient $c_{f}$ versus the streamwise direction $x$ for a TS wavepacket without control (solid black line) and with control (lines with markers, see the legend). The parameters of the controlled cases are reported in table 3.

the actuation array, the perturbation gradually recovers its spanwise coherence, which leads to renewed growth of the TS wavepacket for both the controlled case $L 9$ and S17.

The skin friction coefficient $c_{f}=\partial u /\left.\partial y\right|_{y=0}$ as a function of the downstream distance is shown in figure 6 ; the $c_{f}$ is quantified by averaging along the spanwise direction and considering the time-averaged mean along $x$ by following the time propagation of the perturbation. The sudden increase of $c_{f}$ indicates the onset of transition (Schmid \& Henningson 2001). The TS wavepacket triggers the onset of transition at $x \approx 1400$. For the compensated cases, we observe that transition is delayed by approximately $\Delta x=300$, corresponding to $R e_{x} \approx 3.0 \times 10^{5}$, when the controller $S 17$ is applied. For the case $L 9, \Delta x=250$, while for $L 17$, the delay is $\Delta x=200$. Thus, the higher disturbance amplitudes observed in figure $5(a)$ for $L 17$ promote earlier transition to turbulence than for $L 9$.

\section{Analysis of the controlled flow}

The three-dimensionality of the localized spanwise arrangement of the actuators introduces new disturbances in the field. Since we are mainly interested in understanding the underlying physics of the new structures introduced, we perform an amplitude expansion of the flow fields at a fixed time. The following expansion is applied to the perturbation velocity field,

$$
\boldsymbol{u}\left(t, a_{0 j}\right)^{\prime}=\sum_{k=1}^{n} a_{0 j}^{k} \tilde{\boldsymbol{u}}_{k}(t)+O\left(a_{0 j}^{n+1}\right), \quad j=1, \ldots, n
$$

Here, $a_{0 j}$ represents the initial amplitudes of the $j$ th perturbation velocity field $\boldsymbol{u}(t)^{\prime}$. The expansion basis is composed by $n$ flow fields $\tilde{\boldsymbol{u}}_{k}(t)$. The first order $n=1$ corresponds to the solution of the linearized system, while $n>1$ is related to perturbations triggered by nonlinear interactions. Neglecting higher-order terms, the 
(a)

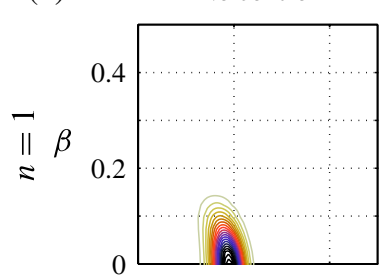

$(d)$

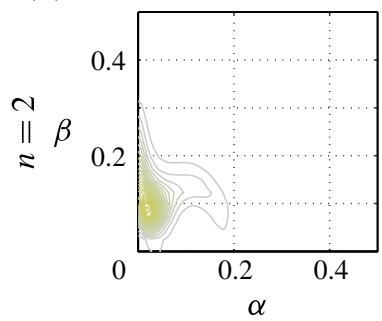

(b)
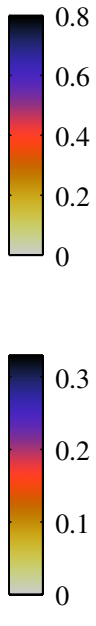

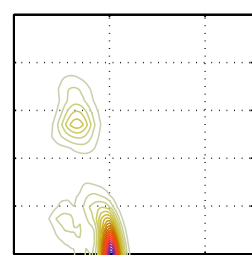

$(e)$

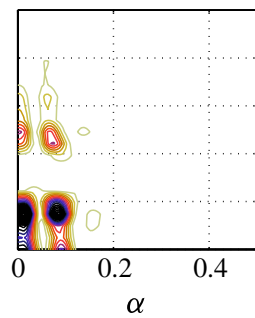

(c) $\quad S 17$

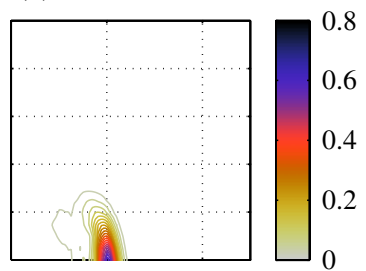

$(f)$

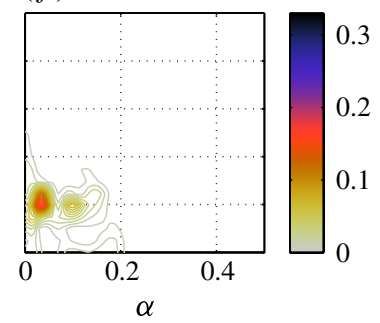

FIgURE 7. Amplitude expansion analysis for TS waves in the $\alpha-\beta$ plane at $t=1000$. The uncontrolled case $(a, d)$ is compared with the controlled case $L 9(b, e)$ and $S 17(c, f)$. The spectrum for the streamwise component $\tilde{u}(t)$ is shown. The order $n=1$ is reported in the first row for all cases, while $n=2$ is shown in the second. The contour levels are normalized to the maximum level in $(a)$, where the maximum amplification is attained.

expansion in (4.1) can be written in matrix form as

$$
\left[\begin{array}{cccc}
\left(a_{01}\right)^{1} & \left(a_{01}\right)^{2} & \ldots & \left(a_{01}\right)^{n} \\
\left(a_{02}\right)^{1} & \left(a_{02}\right)^{2} & \ldots & \left(a_{02}\right)^{n} \\
\ldots & \ldots & \ddots & \ldots \\
\left(a_{0 n}\right)^{1} & \left(a_{0 n}\right)^{2} & \ldots & \left(a_{0 n}\right)^{n}
\end{array}\right]\left[\begin{array}{c}
\tilde{\boldsymbol{u}}_{1}(t) \\
\tilde{\boldsymbol{u}}_{2}(t) \\
\ldots \\
\tilde{\boldsymbol{u}}_{n}(t)
\end{array}\right]=\left[\begin{array}{c}
\boldsymbol{u}\left(t, a_{01}\right)^{\prime} \\
\boldsymbol{u}\left(t, a_{02}\right)^{\prime} \\
\ldots \\
\boldsymbol{u}\left(t, a_{0 n}\right)^{\prime}
\end{array}\right]
$$

The flow fields $\tilde{\boldsymbol{u}}_{k}(t)$ are the unknowns of the linear system and represent the expansion-basis at a given time $t$. The column-vector on the right-hand side consists of $n$ velocity fields $\boldsymbol{u}\left(t, a_{0 j}\right)^{\prime}$, computed by running several simulations with different values of $a_{0}$ (see Henningson, Lundbladh \& Johansson 1993, for more details).

We choose $n=4$, with amplitudes defined at the initial instant $t=0$ in the interval $a_{0}=\{0.20,5.0\} \times 10^{-2} \%$; the spatial location of the packet corresponds along the streamwise direction at $x_{w} \approx 50$. This range of amplitudes corresponds to the range $a_{\text {ref }}=\{0.05,0.22\} \%$ at the actuator location (for the uncontrolled configuration). In the following, we analyse the linear part $n=1$ and the nonlinear interactions $n=2$. The focus is on the analysis of those interactions that are responsible for the deviation from the linear behaviour and the worsening of performance of the controller. In figure 7 , the streamwise velocity component is analysed at $t=1000$ in the $\alpha-\beta$ plane for the first two orders $n$ for the uncontrolled case and the controlled cases based on the configuration $L 9$ and $S 17 ; \alpha=2 \pi N_{x} / L_{x}$ is the streamwise wavenumber and $\beta=2 \pi N_{z} / L_{z}$ the spanwise wavenumber of the perturbation. The order $n=1$ is further investigated in figure 8, where a map in spectral space of the maximum amplitude over $\alpha$ is analysed as a function of time $t$ and the wavenumber $\beta$. 
(a)

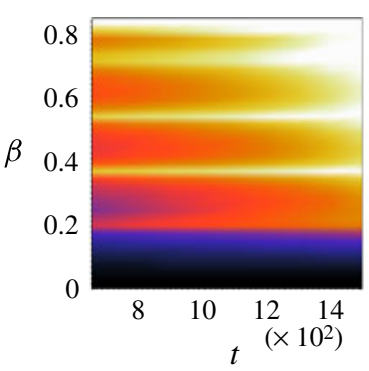

(b)

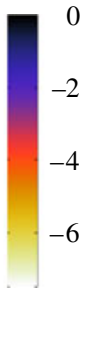

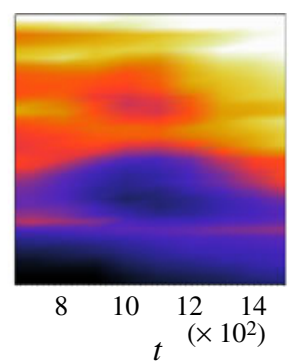

(c)

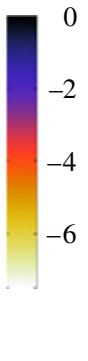

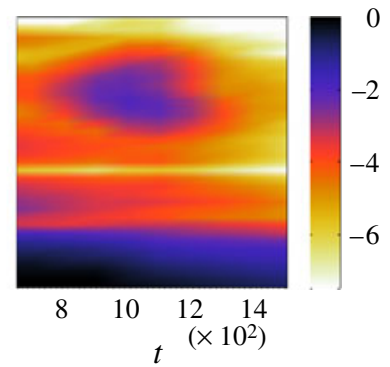

FIGURE 8 . Linear order $n=1$ in the $t-\beta$ plane. The maximum amplitude of the streamwise component $\tilde{u}(t)$ over $\alpha$ is chosen for each $(\beta, t)$ : $(a)$ uncontrolled case; $(b)$ case $L 9 ;(c)$ case $S 17$. The scale of the contour level is logarithmic; the amplitude values are scaled with the maximum amplification of the uncontrolled case (maximum level in figure $8 a$ ).

First, the uncontrolled flow is considered at $t=1000$ in figure $7(a, d)$. The first order $n=1$ is dominated by the TS frequency $\alpha=0.2$ (figure 7a). The quadratic nonlinear interaction, $n=2$, introduces structures elongated in the streamwise direction and characterized by $\beta \approx 0.1$ (figure $7 d$ ).

The controller $L 9$ is analysed in figures $7(b, e)$ and $8(b)$. As discussed in the previous section, the three-dimensionality of the control action introduces new wavenumber characteristics of the controller. Indeed, the spectrum of the first-order term (figure $7 b$ ) in the expansion shows a clear damping of the energy at the frequency related to the TS waves and the appearance of three-dimensional modes due to the localization of the actuation. The first evidence of the controller footprint appears at $t \approx 800$, with a spanwise wavenumber $\beta_{a c t} \approx 0.3$ (figure $8 b$ ). At this instant of time, the leading edge of the wavepacket approaches the actuator array at $x_{\phi}=400$. Note that this mode is related to the distribution of the actuators along the spanwise direction. Indeed $\beta_{a c t}=2 \pi / \Delta z \approx 0.3$, where $\Delta z=20$; the streamwise wavenumber, $\alpha \approx 0.14$ (see figure $7 b$ ) is related to the streamwise wavelength of the perturbation produced by the actuators. At a later time, we see the interaction with the wavepacket, producing a damping of the amplitudes related to the TS wavepacket. The amplitude of threedimensional mode increases in the interval $t \in[800,1400]$ until it disappears once the trailing edge of the wavepacket has propagated downstream of the actuator row $\boldsymbol{B}_{2}$, as shown in figure $8(b)$.

The presence of the three-dimensional mode at the order $n=1$ strongly affects the higher-order terms. Indeed, the quadratic term $n=2$ shows two peaks at $\beta \approx 0.1$ (figure 7e); the first is characterized by a lower value of $\alpha$ and is related to the nonlinearities of the uncontrolled flow. The second peak is related to the interaction between the action of the controller and the uncontrolled flow. Moreover, it is observed that in the nonlinear terms $n>1$, higher-order spanwise modes $\beta>0.2$ appear. Also this region can be related to the geometry of the actuator adopted in L9; thus, the propagation of energy to smaller spanwise scales appears to be due to the interaction between the controller and the uncontrolled flow. This worsens the performance of the controller for larger amplitudes of the initial disturbance and reduces the robustness of the controller when a stronger controller effort is introduced. Also, note that for the controller $L 9$ the scales at $n=2$ is one order of magnitude larger than the uncontrolled case, see figure 7(e). 
As already argued from the analysis of the instantaneous flow and the behaviour of the perturbation amplitude $a(t)$ in figure 5, a different set-up can lead to substantial improvements in the control action. The same analysis is now carried out for the case $S 17$, characterized by smaller actuators, $m=17$ and $\Delta z=10$. In figures $7(c, f)$ and $8(c)$, the behaviour of the controller $S 17$ is described by following the same steps undertaken in the previous paragraph. At order $n=1$, the controller $S 17$ generates a three-dimensional mode at $\beta_{a c t} \approx 0.6$. The mode is depicted in $8(c)$, where the spectral analysis is shown by using logarithmic contour levels in the $t-\beta$ plane. The mode related to the controller $S 17$ is characterized by smaller amplitude over all the interval of action, if compared with the controller $L 9$. Indeed, the maximum amplitude for this mode is attained at $t=1100$ and it is around two or three orders of magnitudes weaker than the maximum amplification of the TS wave.

The interactions appearing now at $n=2$ are mostly related to the action of the controller on the TS wave combined with the nonlinearities of the underlying flow. The maximum amplitude of the modes at $n=2$ for the controller $S 17$ is $75 \%$ less than the $L 9$ case and closer to the level of the original flow, as clearly shown by the direct comparison of figure $7(e, f)$.

\section{Conclusions}

We have demonstrated the possibility to delay the transition using an active controller based on localized sensors/actuators by using numerical simulations. Threedimensional TS wavepackets with finite amplitudes have been considered; ROMs based on balanced realization have been built and used for the design of optimal controllers. The controller mitigates the disturbances amplitude when the nonlinear flow is still laminar, resulting in a significant reduction of the perturbation energy and, later, in a delay of the transition process.

LQG control design has been applied in combination with balanced realizations of the linearized Navier-Stokes system. Due to the strong time delays characterizing the system and the placement of the actuators and sensors, from the dynamical point of view the closed-loop system corresponds to a disturbance feedforward controller, a special case of output feedback control (see, e.g., Anderson \& Liu 1989). This configuration guarantees the best performance in terms of minimization of the norm $\left\|\boldsymbol{z}^{\prime}\right\|$ and damping of the perturbation energy amplitude (see Belson et al. 2013).

A parametric analysis of the performance has been carried out by varying the controller effort and the finite amplitude of the perturbation for three different configurations of actuators. The actuators' array consist of localized elements, distributed along the spanwise direction; each element is defined by a solenoidal Gaussian-like function and different size for the spatial distribution of the forcing were tested. A significant improvement has been found when actuators with a smaller spatial distribution and a shorter distance among the elements of the array are used; in this case, a good agreement with the linear prediction is observed below an amplitude $a<0.1 \%$ at the actuator location. The controllers designed using this configuration are effective in delaying the transition to turbulence in presence of perturbation with amplitudes up to $a \approx 1 \%$ at the actuator location.

We have also highlighted limitations related to the set-up. The localized actuation introduces secondary instability modes that might promote transition to turbulence. This effect is due to the excitation of higher-order harmonics, related to the threedimensionality of the controller action, combined with the nonlinearities of the uncontrolled flow. This issue becomes particularly relevant when strong controller 
efforts are employed, i.e. for low values of the control penalty; in such conditions, the behaviour of the controller quickly deviates from the linear prediction when higher amplitudes are considered.

Based on this knowledge, a further improvement of the device can be achieved by explicitly accounting for the nonlinear effects during the modelling process. Modern developments in robust control theory may be used to rigourously incorporate uncertainties in the design process during the modelling step (see, e.g., Hervé et al. 2012) or by introducing adaptive controllers (see, e.g., Sturzebecher \& Nitsche 2003). The parametric analysis proposed in this work clearly suggests that a careful analysis of the actuation, based on both linear and nonlinear simulations, is helpful to determine the main parameters characterizing the geometry of a fully threedimensional configuration.

\section{Acknowledgements}

Computer time provided by SNIC (Swedish National Infrastructure for Computing) and financial support from the Swedish Research Council (VR) are gratefully acknowledged.

\section{Appendix. Control design}

The control design adopted in this work is based on the reduce-then-design scheme. Following this approach, the first step consists of building a ROM of the system. The controller is designed using this model and applied in the numerical simulations.

This methodology is particularly convenient for large dynamical systems; indeed, the system in (2.2) obtained by discretizing the linearized Navier-Stokes equations is characterized by a number of degree of freedom of order $\approx 10^{7}-10^{8}$. However, among all of the possible flow disturbances, only a portion can be excited by the inputs and observed by the outputs; in literature, these states are referred to as controllable and observable (see e.g. Skogestad \& Postlethwaite 2005). When $m, p \ll n$, most part of the states is scarcely controllable and observable due to the limited number of inputs and outputs; thus, it is possible to accurately reproduce the input-output behaviour while disregarding these uncontrollable/unobservable states. Balanced truncation provides a systematic way for performing such a reduction (Moore 1981): the state vector of the system is transformed such that each element is equally controllable and observable. Moreover, we rank the states from the most controllable/observable to the least controllable/observable; the rank of the states allows to truncate the states that do not influence strongly the input-output dynamics of the system.

Balanced realizations can be obtained by using a system identification algorithm referred to in the literature as ERA, see Juang \& Pappa (1985). This algorithm is an approximation of the balanced truncation; we briefly outline the main steps in the following. First, we compute the Markov parameters of the system by simulating the impulse response in each input $\boldsymbol{B}_{i}$; by defining the propagator operator $\boldsymbol{T}=\mathrm{e}^{\boldsymbol{A \Delta t}}$, we get

$$
\left[\begin{array}{lllll}
\boldsymbol{C B} & \boldsymbol{C T} \boldsymbol{B} & \boldsymbol{C} \boldsymbol{T}^{2} \boldsymbol{B} & \ldots & \boldsymbol{C} \boldsymbol{T}^{m_{c}+m_{0}+1} \boldsymbol{B}
\end{array}\right],
$$


where $\boldsymbol{B} \in \mathbb{R}^{n \times(d+m)}$ and $\boldsymbol{C} \in \mathbb{R}^{(k+p) \times n}$ are defined as

$$
\boldsymbol{B}=\left[\begin{array}{ll}
\boldsymbol{B}_{1} & \boldsymbol{B}_{2}
\end{array}\right], \quad \boldsymbol{C}=\left[\begin{array}{l}
\boldsymbol{C}_{1} \\
\boldsymbol{C}_{2}
\end{array}\right] .
$$

The parameter $d$ indicates the number of disturbance, while the quantities $m, k$ and $p$ were already defined in the $\S 2$. The terms $\boldsymbol{C} \boldsymbol{A}^{j} \boldsymbol{B} \in \mathbb{R}^{(k+p) \times(d+m)}$ are commonly called Markov parameters; in (A 1), the Markov parameters are stacked at a sampling time $t_{j}=M \Delta t$, where $M$ is an integer number.

The sequence of Markov parameters can be arranged into two Hankel matrices. The first matrix is indicated as $\boldsymbol{H}_{0} \in \mathbb{R}^{m_{o} \times m_{c}}$ and reads

$$
\boldsymbol{H}_{0}=\left[\begin{array}{llll}
\boldsymbol{C B} & \boldsymbol{C} \boldsymbol{T} \boldsymbol{B} & \ldots & \boldsymbol{C} \boldsymbol{T}^{m_{c}} \boldsymbol{B} \\
\boldsymbol{C} \boldsymbol{T} \boldsymbol{B} & \boldsymbol{C} \boldsymbol{T}^{2} \boldsymbol{B} & \ldots & \boldsymbol{C} \boldsymbol{T}^{m_{c}+1} \boldsymbol{B} \\
\vdots & \vdots & \ddots & \vdots \\
\boldsymbol{C} \boldsymbol{T}^{m_{o}} \boldsymbol{B} & \boldsymbol{C} \boldsymbol{T}^{m_{o}+1} \boldsymbol{B} & \ldots & \boldsymbol{C} \boldsymbol{T}^{m_{c}+m_{o}} \boldsymbol{B}
\end{array}\right]
$$

The second Hankel matrix $\boldsymbol{H}_{1} \in \mathbb{R}^{m_{o} \times m_{c}}$ is obtained from $\boldsymbol{H}_{0}$ by cutting the row $r=1$ and adding an extra row of Markov parameters

$$
\left[\begin{array}{llll}
\boldsymbol{C} \boldsymbol{T}^{m_{o}+1} \boldsymbol{B} & \boldsymbol{C} \boldsymbol{T}^{m_{o}+2} \boldsymbol{B} & \ldots & \boldsymbol{C} \boldsymbol{T}^{m_{c}+m_{o}+1} \boldsymbol{B}
\end{array}\right],
$$

at $r=m_{o}$. In general, these block matrices are rectangular and characterized by constant skew-diagonals. A singular value decomposition (SVD) is performed such that

$$
\boldsymbol{H}_{0}=\boldsymbol{U} \boldsymbol{\Sigma} \boldsymbol{V}^{H} .
$$

The Hankel singular values (HSV) are contained in the diagonal matrix $\boldsymbol{\Sigma} \in \mathbb{R}^{m_{o} \times m_{c}}$; the superscript $(\cdot)^{H}$ indicates the transpose matrix. We keep the first $r$ states, by considering the first $r$ columns of the matrices $\boldsymbol{U} \in \mathbb{R}^{m_{o} \times m_{o}}$, the first $r$ rows of the matrix $\boldsymbol{V} \in \mathbb{R}^{m_{c} \times m_{c}}$, and the first $r$ rows and columns of $\boldsymbol{\Sigma}$. The resulting matrices $\boldsymbol{U}_{r} \in \mathbb{R}^{m_{o} \times r}, \boldsymbol{V}_{r} \in \mathbb{R}^{m_{c} \times r}$ and $\boldsymbol{\Sigma}_{r} \in \mathbb{R}^{r \times r}$ are used for finding the ROM of the system; indeed, the ROM is identified by the matrices $\boldsymbol{A}_{r} \in \mathbb{R}^{r \times r}, \boldsymbol{B}_{r} \in \mathbb{R}^{r \times(d+m)}$ and $\boldsymbol{C}_{r} \in \mathbb{R}^{(k+p) \times r}$ defined as

$$
\begin{gathered}
\boldsymbol{A}_{r}=\boldsymbol{\Sigma}_{r}^{-1 / 2} \boldsymbol{U}_{r}^{H} \boldsymbol{H}_{1} \boldsymbol{V}_{r} \boldsymbol{\Sigma}_{r}^{-1 / 2}, \\
\boldsymbol{B}_{r}=\text { the first }(d+m) \text { columns of } \boldsymbol{\Sigma}_{r}^{-1 / 2} \boldsymbol{V}_{r}, \\
\boldsymbol{C}_{r}=\text { the first }(k+p) \text { rows of } \boldsymbol{U}_{r} \boldsymbol{\Sigma}_{r}^{-1 / 2} .
\end{gathered}
$$

The equivalence of this procedure with balanced truncation can be shown by directly comparing the relations in A.6 with ROM obtained as projection onto a base of balanced modes (see, for more details, Ma et al. 2011). The resulting ROM is in time-discrete form, but it can easily converted in continuous-time form (Glad \& Ljung 2001) for running the ROM next to the main DNS simulation.

The size of the models is chosen by considering the HSV. Indeed, using the HSV, it is possible to evaluate a priori the theoretical error bounds when exact balanced truncation is performed (see Skogestad \& Postlethwaite 2005). By assuming that the theoretical bounds of the exact balanced truncation are valid also for the approximate case when the converged modes are considered (see Bagheri et al. 2009b), we kept an error bound of order $O\left(10^{-4}\right)$ for all of the models used in this work. 
The controller is designed using the LQG approach on the low-order model of dimension $r$. The design of the controller is split in two steps. For a complete derivation of the LQG solution, we refer to Lewis \& Syrmos (1995), Dullerud \& Paganini (1999) and Bagheri et al. (2009b). First, the controller is computed by assuming full knowledge of the state and solving the associated algebraic Riccati equation

$$
0=\boldsymbol{A}_{r}^{H} \boldsymbol{X}+\boldsymbol{X} \boldsymbol{A}_{r}-\boldsymbol{X} \boldsymbol{B}_{2 r} \boldsymbol{R}^{-1} \boldsymbol{B}_{2 r}^{H} \boldsymbol{X}+\boldsymbol{C}_{1 r}^{H} \boldsymbol{C}_{1 r} .
$$

The Riccati equation results from the optimization problem based on the minimization of the quadratic cost function in (2.4) applied on the ROM; the underlying hypothesis is the time invariance of the system, i.e. we assume steady solution for $T \rightarrow \infty$. The symmetric matrix $\boldsymbol{X} \in \mathbb{R}^{r \times r}$ is used for computing the control gain $\boldsymbol{K} \in \mathbb{R}^{m \times r}$ as

$$
\boldsymbol{K}=-\boldsymbol{R}^{-1} \boldsymbol{B}_{2 r}^{H} \boldsymbol{X},
$$

where the diagonal entries of the matrix $\boldsymbol{R} \in \mathbb{R}^{m \times m}$ contain the control penalty $l^{2}$.

The second step is the estimation. A dynamic system referred to as an estimator is defined

$$
\begin{aligned}
\dot{\hat{\boldsymbol{u}}}_{r}(t) & =\boldsymbol{A}_{r} \hat{\boldsymbol{u}}_{r}(t)+\boldsymbol{B}_{1 r} \boldsymbol{w}(t)+\boldsymbol{B}_{2 r} \phi(t)-\boldsymbol{L}(\psi(t)-\hat{\psi}(t)), \\
\psi(t) & =\boldsymbol{C}_{2} \boldsymbol{u}(t)^{\prime}+\eta \boldsymbol{g}(t), \\
\hat{\psi}(t) & =\boldsymbol{C}_{2 r} \hat{\boldsymbol{u}}_{r}(t) .
\end{aligned}
$$

The unknown estimation gain $\boldsymbol{L} \in \mathbb{R}^{r \times p}$ drives the system in (A $9 a$ ) such that the error norm $\left\|\boldsymbol{u}_{r}-\hat{\boldsymbol{u}}_{r}\right\|^{2}$ is minimized. Thus, a second optimization can be cast, whose solution is provided by a second Riccati equation

$$
0=\boldsymbol{A}_{r} \boldsymbol{Y}+\boldsymbol{Y} \boldsymbol{A}_{r}^{H}-\boldsymbol{Y} \boldsymbol{C}_{2 r}^{H} \boldsymbol{G}^{-1} \boldsymbol{C}_{2 r} \boldsymbol{Y}+\boldsymbol{B}_{1 r} \boldsymbol{W} \boldsymbol{B}_{1 r}^{H} .
$$

Using the matrix $\boldsymbol{Y} \in \mathbb{R}^{r \times r}$, the estimation gain $\boldsymbol{L} \in \mathbb{R}^{r \times p}$ assumes the form

$$
\boldsymbol{L}=-\boldsymbol{Y} \boldsymbol{C}_{2 r}^{H} \boldsymbol{G}^{-1} .
$$

Note that the matrices $\boldsymbol{G} \in \mathbb{R}^{p \times p}$ and $\boldsymbol{W} \in \mathbb{R}^{d \times d}$ are related to the covariances of the input signals $\boldsymbol{g}(t)$ and $\boldsymbol{w}(t)$, respectively. The final output feedback controller or compensator is obtained by combining the estimator and the controller; for the separation principle, it can be shown that the two optimal solutions provide an optimal compensator, guaranteed to be stable.

A final remark is related to the MIMO configuration. We followed the centralized control approach, where all of the $m$ actuators $\boldsymbol{B}_{2}$ are connected to all of the $p$ sensors $\boldsymbol{C}_{2}$. The centralized control consists of only one control-unit based on the control gain $\boldsymbol{K} \in \mathbb{R}^{m \times r}$ and estimation gain $\boldsymbol{L} \in \mathbb{R}^{r \times p}$. A second approach is the socalled decentralized control; in the simplest case, each sensor $\boldsymbol{C}_{2}$ is connected to only one actuator $\boldsymbol{B}_{2}$ (Glad \& Ljung 2001; Skogestad \& Postlethwaite 2005). Following this approach, a number of control units that equals the number of sensor/actuator pairs, $m=p$, is designed; despite the relative simplicity of the design, particularly advantageous in practical implementations, the choice is prone to stability problems that is not guaranteed in the closed loop. This is the case of our configuration; indeed, the described decentralized approach was found to be unstable for the TS wave controller (see Semeraro et al. 2011). Similar considerations are presented by Li \& Gaster (2006). 


\section{REFERENCES}

Adams, N. \& Stolz, S. 1999 On the approximate deconvolution procedure for LES. Phys. Fluids 2, 1699-1701.

Åkervik, E., Hepffiner, J., Ehrenstein, U. \& Henningson, D. S. 2007 Optimal growth, model reduction and control in a separated boundary-layer flow using global eigenmodes. J. Fluid. Mech. 579, 305-314.

ANDERSON, B. \& LiU, Y. 1989 Controller reduction: concepts and approaches. IEEE Trans. Autom. Control 34, 802-812.

Andersson, P., Berggren, M. \& Henningson, D. 1999 Optimal disturbances and bypass transition in boundary layers. Phys. Fluids 11, 134-150.

Andersson, P., Brandt, L., Bottaro, A. \& Henningson, D. 2001 On the breakdown of boundary layer streaks. J. Fluid Mech. 428, 29-60.

BAgheri, S., BRANDT, L. \& Henningson, D. S. $2009 a$ Input-output analysis, model reduction and control design of the flat-plate boundary layer. J. Fluid Mech. 620, 263-298.

Bagheri, S. \& Henningson, D. S. 2011 Transition delay using control theory. Phil. Trans. R. Soc. A 369, 1365-1381.

Bagheri, S., Hepffner, J., Schmid, P. J. \& Henningson, D. S. $2009 b$ Input-output analysis and control design applied to a linear model of spatially developing flows. Appl. Mech. Rev. 62 (2), 020803.

Barbagallo, A., Sipp, D. \& Schmid, P. 2009 Closed-loop control of an open cavity flow using reduced order models. J. Fluid Mech. 641, 1-50.

Belson, B., Semeraro, O., Rowley, C. \& Henningson, D. 2013 Feedback control of instabilities in the two-dimensional Blasius boundary layer: the role of sensors and actuators. Phys. Fluids 25, 054106.

BEWLEY, T. R. \& LiU, S. 1998 Optimal and robust control and estimation of linear paths to transition. J. Fluid Mech. 365, 305-349.

Brandt, L., Sipp, D., Pralits, J. O. \& Marquet, O. 2011 Effect of base-flow variation in noise amplifiers: the flat-plate boundary layer. J. Fluid Mech. 687, 503-528.

Chevalier, M., Schlatter, P., Lundbladh, A. \& Henningson, D. S. 2007 A pseudo spectral solver for incompressible boundary layer flows. In Trita-Mek 7. KTH Mechanics, Stockholm, Sweden.

Cortelezzi, L., Speyer, J. L., Lee, K. H. \& KiM, J. 1998 Robust reduced-order control of turbulent channel flows via distributed sensors and actuators. In IEEE 37th Conference of Decision Control, pp. 1906-1911.

Doyle, J. C., Glover, K., Khargonekar, P. P. \& Francis, B. A. 1989 State-space solutions to standard $\mathrm{H}_{2}$ and $\mathrm{H}_{\infty}$ control problems. IEEE Trans. Autom. Control 34, 831-847.

Dullerud, E. G. \& Paganini, F. 1999 A Course in Robust Control Theory. A Convex Approach. Springer.

Glad, T. \& LJung, L. 2001 Control Theory - Multivariable and Nonlinear Methods. Taylor and Francis.

Grundmann, S. \& Tropea, C. 2008 Active cancellation of artificially introduced Tollmien-Schlichting waves using plasma actuators. Exp. Fluids 44 (5), 795-806.

Henningson, D. S., Lundbladh, A. \& Johansson, A. V. 1993 A mechanism for bypass transition from localized disturbances in wall-bounded shear flows. J. Fluid Mech. 250, 169-207.

Hervé, A., Sipp, D., Schmid, P. J. \& SAmuelides, M. 2012 A physics-based approach to flow control using system identification. J. Fluid Mech. 702, 26-58.

IlAK, M. \& Rowley, C. W. 2008 Modelling of transitional channel flow using balanced proper orthogonal decomposition. Phys. Fluids 20, 034103.

Joshi, S. S., Speyer, J. L. \& KIM, J. 1997 A systems theory approach to the feedback stabilization of infinitesimal and finite-amplitude disturbances in plane Poiseuille flow. J. Fluid Mech. 332, 157-184.

JUANG, J. \& PAPPA, R. S. 1985 An eigensystem realization algorithm for modal paramter identification and model reduction. J. Guid. Control. Dyn. 3 (25), 620-627. 
Juillet, F., Schmid, P. J. \& Huerre, P. 2013 Control of amplifier flows using subspace identification techniques. J. Fluid Mech. 725, 522-565.

Kachanov, Y. S. 1994 Physical mechanisms of laminar-boundary-layer transition. Annu. Rev. Fluid Mech 26 (1), 411-482.

Kim, J. \& Bewley, T. R. 2007 A linear systems approach to flow control. Annu. Rev. Fluid Mech. 39, 383-417.

Kriegseis, J. 2011 Performance characterization and quantification of dielectric barrier discharge plasma actuators. PhD thesis, TU Darmstadt.

Lewis, F. L. \& Syrmos, L. V. 1995 Optimal Control. John Wiley and Sons.

LI, Y. \& GASTER, M. 2006 Active control of boundary-layer instabilities. J. Fluid Mech. 550, $185-205$.

LUNDELL, F. 2007 Reactive control of transition induced by free stream turbulence: an experimental demonstration. J. Fluid Mech. 585, 41-71.

Ma, Z., Ahuja, S. \& Rowley, C. W. 2011 Reduced order models for control of fluids using the Eigensystem Realization Algorithm. Theoret. Comput. Fluid Dyn. 25 (1), 233-247.

Monokrousos, A., Åkervik, E., Brandt, L. \& Henningson, D. S. 2010 Global optimal disturbances in the Blasius boundary-layer flow using time steppers. J. Fluid Mech. 650, $181-214$.

Monokrousos, A., Brandt, L., Schlatter, P. \& Henningson, D. S. 2008 DNS and LES of estimation and control of transition in boundary layers subject to free stream turbulence. Intl $J$. Heat Fluid Flow 29 (3), 841-855.

MoORE, B. 1981 Principal component analysis in linear systems: controllability, observability, and model reduction. IEEE Trans. Automat. Control 26 (1), 17-32.

Nordström, J., Nordin, N. \& Henningson, D. S. 1999 The fringe region technique and the Fourier method used in the direct numerical simulation of spatially evolving viscous flows. SIAM J. Sci. Comput. 20 (4), 1365-1393.

Rowley, C. W. 2005 Model reduction for fluids using balanced proper orthogonal decomposition. Intl J. Bifurcation Chaos 15 (3), 997-1013.

Rowley, C. \& Juttiundata, W. 2005 Model-based control and estimation of cavity flow oscillations. In 44th IEEE Conference on Decision and Control, pp. 512-517.

Schlatter, P., Stolz, S. \& Kleiser, L. 2004 LES of transitional flows using the approximate deconvolution model. Intl J. Heat Fluid Flow 25 (3), 549-558.

Schmid, P. J. 2007 Nonmodal stability theory. Annu. Rev. Fluid Mech. 39, 129-162.

Schmid, P. J. \& Henningson, D. S. 2001 Stability and Transition in Shear Flows. Springer.

Semeraro, O., Bagheri, S., Brandt, L. \& Henningson, D. S. 2011 Feedback control of three-dimensional optimal disturbances using reduced-order models. J. Fluid Mech. 677, 63-102.

Sipp, D., Marquet, O., Meliga, P. \& Barbagallo, A. 2010 Dynamics and control of global instabilities in open-flows: a linearized approach. Appl. Mech. Rev. 63 (3), 30801.

Skogestad, S. \& Postlethwaite, I. 2005 Multivariable Feedback Control, Analysis to Design, 2nd edn. Wiley.

Sturzebecher, D. \& Nitsche, W. 2003 Active cancellation of Tollmien-Schlichting waves instabilities on a wing using multi-channel sensor actuator systems. Intl J. Heat Fluid Flow 24, 572-583. 